\title{
Neoadjuvant Weekly Paclitaxel-Carboplatin Is Effective in Stage I-II Cervical Cancer
}

\author{
Rawand Salihi, MD, * Karin Leunen, MD, PhD, * Philippe Moerman, MD, PhD, $\dagger$ \\ Frédéric Amant, $M D, P h D, \neq$ Patrick Neven, $M D, P h D, *$ and Ignace Vergote, $M D, P h D^{*}$
}

\begin{abstract}
Objective: Neoadjuvant chemotherapy (NACT) followed by surgery in cervical cancer is widely studied with paclitaxel-ifosfamide-cisplatinum 3 weekly (TIP). Although the response rates with TIP are high, the toxicity is substantial. Therefore, this study evaluates dose-dense paclitaxel-carboplatin (TC) as an alternative.

Methods: In this prospective phase 2 study trial, we included 36 patients with International Federation of Gynecology and Obstetrics (FIGO) stage IB1 to IIB cervical cancer, who received 9 weeks' NACT dose-dense TC (median weekly dose paclitaxel $60 \mathrm{mg} / \mathrm{m}^{2}$, carboplatinum area under the curve 2.7). Radiological response was evaluated by RECIST (Response Evaluation Criteria in Solid Tumors). Optimal pathologic response (OPT) was defined as complete disappearance of tumor (complete response [CR]) or residual disease with less than 3-mm stromal invasion (PR1). Suboptimal pathologic response consisted of persistent residual disease with more than 3-mm stromal invasion (PR2).

Results: Nine patients had a FIGO stage IB1 (25\%), 7 had stage IB2(19\%), 3 had stage IIA $(8 \%)$, and 17 had stage IIB disease (47\%). Evaluation by magnetic resonance imaging after NACT showed 32 RECIST responses (89\%) (CR in 11, PR in 21). Patients who were inoperable had insufficient reduction of the tumor to be operable (4 patients), progressive disease (1 patient), or stable disease (1 patient). Thirty patients were suitable for surgery after NACT. Pathology showed OPT in 50\% (CR in 10, PR1 in 5). Thirteen patients had pathologic lymph nodes on radiological evaluation before start of chemotherapy. After chemotherapy, the lymph nodes were negative in $6(47 \%)$ of these patients (pathologic complete remission). Postoperative chemoradiotherapy was administered in 11 patients ( 2 because of close resection margins, 5 because of metastatic lymph node after surgery, 2 because of close resection margins and metastatic lymph nodes after surgery, and 1 tumor $>4 \mathrm{~cm}$ after NACT). Hematologic toxicity was acceptable with no febrile neutropenia and a low nonhematologic toxicity. The estimated 5-year overall survival was $70.8 \%$.

Conclusions: Neoadjuvant TC dose-dense in cervical carcinoma has a high response rate, comparable with TIP, and an acceptable toxicity.
\end{abstract}

Key Words: Cervical cancer, Neoadjuvant chemotherapy, Weekly paclitaxel-carboplatin

Received December 19, 2016, and in revised form January 22, 2017.

Accepted for publication March 10, 2017.

(Int J Gynecol Cancer 2017;00: 00-00)

*Division of Gynaecologic Oncology, Department of Obstetrics and Gynaecology, and $†$ Department of Pathology, Leuven Cancer Institute, University Hospitals Leuven, KU Leuven, Belgium; and †Department

Copyright (C) 2017 by IGCS and ESGO

ISSN: $1048-891 \mathrm{X}$

DOI: $10.1097 / \mathrm{IGC} .0000000000001021$ of Gynaecologic Oncology, Antoni van Leeuwenhoek-Netherlands Cancer Institute, Amsterdam, the Netherlands.

Address correspondence and reprint requests to Rawand R. Salihi, MD, Division of Gynaecologic Oncology, Department of Obstetrics and Gynaecology, Leuven Cancer Institute, University Hospitals Leuven, KU Leuven, Herestraat 49, B-3000 Leuven, European Union. E-mail: rawand.salihi@uzleuven.be.

The authors declare no conflicts of interest. 
E arly-stage cervical cancer (IA1-IB1) can be treated by

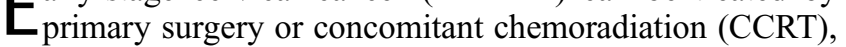
whereas International Federation of Gynecology and Obstetrics (FIGO) stage IIIA or higher is usually treated with CCRT. For locally advanced cervical cancer (FIGO stage IB2-IVA), the most often used therapy is CCRT, but there is evidence that neoadjuvant chemotherapy (NACT) followed by radical hysterectomy $(\mathrm{RH})$ might be an alternative for FIGO stage IB2 to IIB cervical cancer. ${ }^{1,2}$

The principle of NACT followed by RH is to reduce the tumor volume and making the tumor operable. The long-term complications after radiotherapy, castration of the patients, the poor control of metastatic disease, and the lack of possibility for CCRT in less developed countries contribute to the use of NACT followed by surgery.

There have been a number of publications on NACT followed by $\mathrm{RH}^{3}{ }^{3}$ The meta-analysis published on NACT followed by RH compared NACT with radiotherapy alone. This study consisted of 5 randomized trials with a total of 872 patients and showed a significant benefit for NACT followed by surgery compared with radiotherapy.

A second part of the meta-analysis compared NACT followed by radiotherapy versus radiotherapy alone, showing a significant survival benefit for short-interval chemotherapy ( $<14$ days) followed by surgery and for the higher dosages of cisplatin $\left(>25 \mathrm{mg} / \mathrm{m}^{2}\right)$. Since the US Food and Drug Administration alert in 1999, radiotherapy in combination with chemotherapy (weekly cisplatin) (CCRT) has become standard of care because of the improved survival results when adding concomitant chemotherapy. ${ }^{4,5}$ The results of the randomized study of the EORTC (European Organisation for Research and Treatment of Cancer) comparing NACT followed by RH versus CCRT are still awaited.

Several chemotherapeutic agents have been tested as NACT in cervical cancer, with cisplatin, paclitaxel, and ifosfamide considered among the most active drugs. ${ }^{6-9}$ An Italian group showed in the SNAP (Studio Neo-Adjuvante Portio) 01 trial that TIP (paclitaxel, ifosfamide, and cisplatin) resulted in a higher response rate than IP, without a statistically significantly different effect on overall survival. ${ }^{10}$ The SNAP-02 trial of Lissoni et al ${ }^{11}$ compared TP with TIP and showed TIP to be the most active ( $25 \%$ vs $43 \%$ pathological optimal response rate). However, TIP also has an important higher morbidity compared with TP (neutropenia in $26 \%$ and $76 \%$, respectively). ${ }^{11}$

Recently, it has been shown that 3-weekly paclitaxelcarboplatin has similar efficacy compared with 3-weekly paclitaxel-cisplatinum in recurrent cervical cancer. ${ }^{12}$ In the meantime, Mori et $\mathrm{al}^{13}$ showed in 2010 that weekly paclitaxel and carboplatin at a dose of paclitaxel $60 \mathrm{mg} / \mathrm{m}^{2}$, carboplatin (area under the curve [AUC] 2), as NACT for cervical cancer, to be promising. These findings together with the meta-analysis showing the importance of using dose-dense regimens ${ }^{4}$ and our encouraging results with dose-dense paclitaxel-carboplatin in the recurrent setting resulted in the current prospective nonrandomized phase 2 trial investigating the role of dosedense paclitaxel-carboplatinum as NACT in cervical cancer. ${ }^{14}$

In this study, we present 36 patients treated with weekly TC for locally advanced cervical cancer (IB2-IIB) followed in case of response by RH or early-stage (IB1) cervical cancer followed by conization.

\section{METHODS}

We started this prospective phase 2 trial to investigate the aim of pathologic complete remission after treatment of neoadjuvant paclitaxel and carboplatin in dose-dense doses. Patients were recruited between 2005 and 2014 in the University Hospitals Leuven in Belgium. A data analysis of all patients treated with neoadjuvant paclitaxel-carboplatin chemotherapy was done between 2005 and 2016 in the University Hospitals Leuven. All these patients were planned to receive NACT followed by surgery. Only patients with squamous cell carcinoma, adenocarcinoma, or adenosquamous carcinoma of the cervix were included. Patients with high-risk histology such as small cell, neuroendocrine, and glassy cell tumors were excluded.

An optimal pathological response of $43 \%$ has been reported with TIP. ${ }^{10,11}$ A sample size of 36 patients was planned to provide for an OPT of $50 \%$, with a more than $90 \%$ power and $95 \%$ confidence intervals of the maximum half width equal to $16 \%$ (confidence interval, 34\%-66\%).

The study was approved by the ethical committee of University Hospitals Leuven (S57619).

At diagnosis, all patients had a pretreatment evaluation with medical history, physical examination including pelvic examination under anesthesia and cystoscopy, preoperative blood test including tumor markers (SCC if squamous histology and CA-125 if adenocarcinoma), magnetic resonance imaging scan of the pelvis, and systemic examination by computed tomography (CT) of the thorax and abdomen or whole-body positron emission tomography-CT.

Chemotherapy was administered in a dose-dense regimen at a mean weekly dose of paclitaxel $60 \mathrm{mg} / \mathrm{m}^{2}$ and carboplatin AUC 2.7 (either at weekly infusions at this dose or paclitaxel 90 $\mathrm{mg} / \mathrm{m}^{2}$, carboplatin (AUC 4) on days 1 and 8 , every 3 weeks. The 2 regimens were given for 9 weeks preoperatively.

After NACT clinical examination of the pelvis, magnetic resonance imaging of the pelvis and $\mathrm{CT}$ of the thorax and abdomen or positron emission tomography-CT was repeated. Response was reported according to the Response Evaluation Criteria in Solid Tumors (RECIST, version 1.1). ${ }^{15}$ Surgery was done 4 weeks after the last course of chemotherapy in patients showing response and who were operable. If the patient was inoperable, chemoradiation was advised.

Pathologic response was defined as in the SNAP-1 trial $^{10}$ as OR, including a complete disappearance of tumor in the cervix with negative pelvic lymph nodes (CR), or a residual disease with less than 3-mm stromal invasion or in situ carcinoma (PR1). Suboptimal response consisted of persistent residual disease with more than 3-mm stromal invasion in the surgical specimen (PR2).

Adjuvant chemoradiation was standard prescribed if pathology showed positive lymph nodes or in case of involved resection margins.

Overall survival and progression-free survival were both calculated by Kaplan-Meier curves. SPSS Statistics version 21 by IBM Corporation (Armonk, NY) was used for the statistical 
calculations. Hematologic and nonhematologic toxicities were evaluated according to the Common Terminology Criteria for Adverse Events (CTCAE) version 3.0 criteria. $^{16}$

\section{RESULTS}

The median age of the patients was 45 years (range, 25-75 years). Most patients received a diagnosis of a squamous carcinoma (29 patients, $81 \%$ ), whereas 5 patients received a diagnosis of an adenocarcinoma (14\%), and 2 patients had an adenosquamous carcinoma (6\%). At start, 13 patients $(36 \%)$ had suspected lymph nodes. Median weight was $64 \mathrm{~kg}$ (range, $45-104 \mathrm{~kg}$ ). Nine patients had a FIGO stage IB1 (25\%), 7 patients had stage IB2 (19\%), 3 patients had stage IIA (8\%), and 17 patients had stage IIB disease (47\%). Twelve patients were treated with the $\mathrm{d} 1,8$ regimen every 3 weeks $(33 \%)$, and 24 patients with the weekly regimen $(67 \%)$. The median number of treatment weeks was 9 (range, 6-9 weeks).

Evaluation according to the RECIST criteria after chemotherapy showed 11 patients with complete remissions $(31 \%), 21$ with partial remissions (58\%), 2 with stable diseases $(6 \%)$, and 2 with progressive diseases (2\%) (Table 1).

TABLE 1. RECIST and pathological response in relation to FIGO stage, surgical margins, and histological type (neoadjuvant dose-dense paclitaxel-carboplatin in cervical cancer)

\begin{tabular}{|c|c|c|c|c|}
\hline $\begin{array}{l}\text { Patient } \\
\text { No. }\end{array}$ & $\begin{array}{l}\text { FIGO } \\
\text { Stage }\end{array}$ & $\begin{array}{c}\text { Response } \\
\text { After } \\
\text { Chemo }\end{array}$ & $\begin{array}{c}\text { Positive Surgical } \\
\text { Margins }\end{array}$ & $\begin{array}{l}\text { Pathologic } \\
\text { Response }\end{array}$ \\
\hline 1 & IB2 & PR & 0 & PR1 \\
\hline 2 & IIB & PR & 0 & PR1 \\
\hline 3 & IIB & PR & - & Inoperable* \\
\hline 4 & IB2 & PR & 0 & PR2 \\
\hline 5 & IIB & PR & 0 & PR2 \\
\hline 6 & IIB & PR & 1 & PR2 \\
\hline 7 & IIA & $\mathrm{SD}$ & 1 & PR2 \\
\hline 8 & IIB & PR & - & Inoperable* \\
\hline 9 & IIB & $\mathrm{SD}$ & - & Inoperable* \\
\hline 10 & IIB & PR & 0 & PR2 \\
\hline 11 & IIA & $\mathrm{CR}$ & 0 & $\mathrm{CR}$ \\
\hline 12 & IIA & PR & 0 & PR2 \\
\hline 13 & IIB & PR & - & Inoperable* \\
\hline 14 & IB2 & $\mathrm{CR}$ & 1 & PR2 \\
\hline 15 & IB1 & $\mathrm{CR}$ & 0 & $\mathrm{CR}$ \\
\hline 16 & IB1 & $\mathrm{CR}$ & 0 & $\mathrm{CR}$ \\
\hline 17 & IB1 & $\mathrm{CR}$ & 0 & $\mathrm{CR}$ \\
\hline 18 & IB1 & $\mathrm{CR}$ & 0 & $\mathrm{CR}$ \\
\hline 19 & IB1 & $\mathrm{CR}$ & 0 & CR \\
\hline 20 & IB1 & $\mathrm{CR}$ & 0 & $\mathrm{CR}$ \\
\hline 21 & IB1 & $\mathrm{CR}$ & 0 & $\mathrm{CR}$ \\
\hline 22 & IB1 & PD & 0 & PR2 \\
\hline 23 & IIB & PR & 0 & PR2 \\
\hline
\end{tabular}

After NACT, 21 patients underwent an RH (58\%), and 9 patients underwent a conization (25\%). Five patients were judged to be inoperable because of no RECIST response or when a partial remission was seen but with insufficient reduction of the tumor in order to be operable. They were treated with chemoradiation (20\%). Pathological CR was seen in 10 patients, 5 patients showed a PR1 (OPT pathological response in $50 \%$ of the patients operated on), and 15 patients had a PR2. In 1 patient, adjuvant radiotherapy was administered after surgery because of close resection margin. Five patients received radiotherapy because of a positive lymph node after surgery, 2 because of close resection margins and metastatic lymph nodes after surgery, 1 because of tumor of more than $4 \mathrm{~cm}$ after NACT, 1 patient with a large tumor before chemotherapy and extended lymphovascular invasion and 1 patient because of young age, with positive lymph node before starting NACT and massive lymphovascular invasion.

Another patient was planned for chemoradiation, but at the start of the radiotherapy, progression with distant metastases was observed. She was further treated with chemotherapy.

The median time from the last chemotherapy to surgery was 32 days (range, 13-45 days).

Grades 3 and 4 neutropenia was seen in 20 patients $(56 \%)$ and is the most observed adverse event. Fifteen patients $(42 \%)$ had grade 3 neutropenia, and 5 patients (14\%) had grade 4 . There were no cases of neutropenic fever. None of the

TABLE 1. (Continued)

\begin{tabular}{|c|c|c|c|c|}
\hline $\begin{array}{l}\text { Patient } \\
\text { No. }\end{array}$ & $\begin{array}{l}\text { FIGO } \\
\text { Stage }\end{array}$ & $\begin{array}{c}\text { Response } \\
\text { After } \\
\text { Chemo }\end{array}$ & $\begin{array}{c}\text { Positive Surgical } \\
\text { Margins }\end{array}$ & $\begin{array}{l}\text { Pathologic } \\
\text { Response }\end{array}$ \\
\hline 24 & IB2 & PD & - & Inoperable* \\
\hline 25 & IIB & PR & 0 & PR2 \\
\hline 26 & IB2 & PR & 0 & PR2 \\
\hline 27 & IIB & PR & 0 & PR1 \\
\hline 28 & IIB & PR & - & Inoperable* \\
\hline 29 & IB2 & PR & 0 & PR2 \\
\hline 30 & IIB & $\mathrm{CR}$ & 0 & CR \\
\hline 31 & IIB & PR & 0 & PR2 \\
\hline 32 & IB1 & $\mathrm{CR}$ & 0 & CR \\
\hline 33 & IIB & PR & 0 & PR2 \\
\hline 34 & IB2 & PR & 0 & PR1 \\
\hline 35 & IIB & PR & 0 & PR1 \\
\hline 36 & IIB & PR & - & PR2 \\
\hline \multicolumn{5}{|c|}{$\begin{array}{l}\text { RECIST response with CR (complete response), PR (partial } \\
\text { esponse), SD (stable disease), PD (progressive disease). Pathologic } \\
\text { esponse with CR (complete remission), PR1 (residual disease with }<3- \\
\text { mm stromal invasion including in situ carcinoma), PR2 (suboptimal } \\
\text { esponse consisted of persistent residual disease with }>3 \text {-mm stromal } \\
\text { nvasion on surgical specimen). Positive surgical margins are described } \\
\text { as } 1 \text {, and negative surgical margins as } 0 \text {. If inoperable and no pathology } \\
\text { was obtained: - } \\
\text { *Patients were judged to be inoperable when no RECIST response } \\
\text { was observed or when a partial remission was seen but with insufficient } \\
\text { eduction of the tumor in order to be operable. }\end{array}$} \\
\hline
\end{tabular}


TABLE 2. Number of patients with adverse events of chemotherapy according to the CTCAE classification, version $3.0^{*}$

\begin{tabular}{lccccc}
\hline & Neutropenia & Thrombocytopenia & Anemia & Neuropathy & Kidney \\
\hline Grade 0 & $8(22 \%)$ & $20(56 \%)$ & $3(8 \%)$ & $30(83 \%)$ & $33(92 \%)$ \\
Grade 1 & $1(3 \%)$ & $13(36 \%)$ & $19(53 \%)$ & $5(14 \%)$ & $3(8 \%)$ \\
Grade 2 & $7(19 \%)$ & $2(6 \%)$ & $10(28 \%)$ & $1(3 \%)$ & $0(0 \%)$ \\
Grade 3 & $15(42 \%)$ & $1(3 \%)$ & $4(11 \%)$ & $0(0 \%)$ & $0(0 \%)$ \\
Grade 4 & $5 \dagger(14 \%)$ & $0(0 \%)$ & $0(0 \%)$ & $0(0 \%)$ & $0(0 \%)$ \\
\hline
\end{tabular}

Described toxicity is worst reported grade per patient during administration of chemotherapy.

*CTCAE version 3.0; available at: http://ctep.cancer.gov/protocolDevelopment/electronic_applications/docs/ctcaev3.pdf.

$\uparrow$ No cases of febrile neutropenia.

patients needed colony-stimulating factors. Grade 3 thrombocytopenia was seen in 1 patient. Grade 3 anemia was seen in 4 patients (11\%), whereas grades 1 and 2 anemia was seen in 22 patients. Two of the patients with grade 3 anemia were treated with erythropoietin in combination with intravenous iron therapy. Grade 2 sensoric neuropathy was seen in only 1 patient. Kidney toxicity grade 1 was seen in 3 patients. No deaths related to chemotherapy were seen (Table 2).

The median follow-up time was 17 months (range, 3-100 months). In that period, 8 patients had progressive disease. The mean time to progression was 16 months. The different sites of recurrence are described in Table 3. The estimated mean overall survival in all patients is 76 months with a $95 \%$ confidence interval of 59 to 91 months. The estimated mean progression-free survival is 67 months, with a $95 \%$ confidence interval of 51 to 84 months. The 5 -year overall survival is $70.8 \%$. The 5-year progression-free survival is $61.8 \%$.

\section{DISCUSSION}

Neoadjuvant chemotherapy for cervical cancer is still debated. Promising results have been reported by many retrospective and several prospective randomized studies, but the results of the randomized controlled EORTC 55994 trial comparing NACT followed by RH versus CCRT in stage IB2-IIB are still eagerly awaited.

The search for effective and well-tolerated chemotherapy regimens for NACT is ongoing. TIP has been shown to be one of the most active regimens but is associated with a high toxicity and even mortality. Additional ifosfamide has an important gonadotoxic effect. TIP was compared with TP and IP in the 2 SNAP trials, with lower hematologic toxicity but also lower response rate for the 2-drug combinations. ${ }^{10,11} \mathrm{TC}$ in a 3-weekly scheme showed promising results in 1 study with a clinical response rate of $95 \% .{ }^{17}$ These results together with the meta-analysis showing the importance of using dosedense regimens ${ }^{4}$ and our results with dose-dense paclitaxelcarboplatin in the recurrent setting ${ }^{14}$ resulted in the start of current prospective trial to investigate the pathologic complete response (CR) and toxicity of dose-dense paclitaxelcarboplatin as NACT in cervical cancer.

In this study, we present our results of paclitaxelcarboplatin in dose-dense regimens. We already showed that TC weekly is an effective regimen in recurrent cervical cancer, with a low toxicity profile. ${ }^{14}$ The most severe toxicity was grade 3-4 neutropenia ( $56 \%$ ), but no febrile neutropenia was observed. Grade 2 sensoric neuropathy was seen in only 1

TABLE 3. Site of recurrence

\begin{tabular}{|c|c|c|c|c|c|c|c|c|c|}
\hline $\begin{array}{l}\text { Patient } \\
\text { No. }\end{array}$ & $\begin{array}{c}\text { Type } \\
\text { of Tumor }\end{array}$ & $\begin{array}{l}\text { FIGO } \\
\text { Stage }\end{array}$ & $\begin{array}{l}\text { RECIST } \\
\text { Response }\end{array}$ & $\begin{array}{c}\text { Type } \\
\text { of Surgery }\end{array}$ & $\begin{array}{l}\text { Size of Tumor } \\
\text { on Pathology }\end{array}$ & $\begin{array}{c}\text { Central } \\
\text { Pelvis }\end{array}$ & $\begin{array}{c}\text { Pelvic } \\
\text { Lymph } \\
\text { Node }\end{array}$ & $\begin{array}{c}\text { Upper } \\
\text { Abdomen }\end{array}$ & $\begin{array}{c}\text { Extra } \\
\text { Abdominal }\end{array}$ \\
\hline 5 & Squamous & IB2 & PR & Wertheim & $3.5 \times 2 \times 1.5$ & 0 & 1 & 0 & 1 \\
\hline 6 & Squamous & IIB & PR & Wertheim & $4 \times 2.6$ & 0 & 0 & 0 & 1 \\
\hline 8 & Adenocarcinoma & IIA & SD & Wertheim & $32 \times 25 \times 17$ & 0 & 0 & 0 & 1 \\
\hline 11 & Squamous & IIB & PR & Wertheim & $11 \times 20 \times 30$ & 1 & 1 & 1 & 1 \\
\hline 14 & Squamous & IIB & PR & Inoperable & - & 1 & 0 & 0 & 0 \\
\hline 16 & Adenosquamous & IB1 & $\mathrm{CR}$ & Conization & NRT & 1 & 0 & 0 & 0 \\
\hline 23 & Squamous & IB1 & PD & Wertheim & $20 \times 25 \times 28$ & 0 & 1 & 0 & 1 \\
\hline 29 & Squamous & IIB & PR & Inoperable & - & 0 & 1 & 1 & 0 \\
\hline
\end{tabular}

RECIST response with CR (complete response), PR (partial response), SD (stable disease), PD (progressive disease). Sites of recurrences are denoted as 1 .

NRT, no residual tumor. 
patient. No alopecia is seen, especially since the use of cold caps in our hospital.

In the current study with dose-dense paclitaxelcarboplatin, we observed excellent clinical (89\%) and pathological response rates in patients operated on (no tumor on pathology or invasion of $<3 \mathrm{~mm}$ in $50 \%$ of the patients). This compared with the $84 \%$ clinical response (CR or PR) seen in TIP. ${ }^{18}$ These results are also comparable with those seen by Mori et $\mathrm{al}^{13}$ ( $87 \%$ objective response rate). ${ }^{13}$

The main difference between our study and that of Mori et $\mathrm{al}^{13}$ is the dose of carboplatin. We used a mean weekly dose of paclitaxel $60 \mathrm{mg} / \mathrm{m}^{2}$ and carboplatin (AUC 2.7) (TCw) or paclitaxel $80 \mathrm{mg} / \mathrm{m}^{2}$ and carboplatin (AUC 4) d1,8 every 3 weeks (TCdd), whereas Mori et $\mathrm{al}^{13}$ used the same paclitaxel dose but a carboplatin dose of AUC 2.0.

The pathologic response rate in our population is comparable to the SNAP-02 trial ${ }^{11}$ with a pathologic response of $43 \%$ with TIP and $25 \%$ with TP, 3 weekly. The SNAP-01 trial showed an optimal response rate of $23 \%$ for IP. We should, however, remark that in our series 9 patients with FIGO stage IB1 tumors were included. Table 1 confirms that the highest response rate radiologically and pathologically is seen in the more prognostically favorable tumors (FIGO stage IB1).

The 5-year overall survival and progression-free survival were $70 \%$ and $66 \%$, respectively. The SNAP trial showed a similar overall survival of $78 \%$ for TIP and $71 \%$ for TP. ${ }^{11}$

We therefore propose that weekly paclitaxel carboplatin should be considered as an alternative to TIP. ${ }^{10,11}$ Using our doses described in this study in combination with the weekly administration, in our experience, we find an intensive continuous dose of paclitaxel and carboplatin, with similar results of effectiveness but with less toxicity and better toleration. ${ }^{14}$

In conclusion, dose-dense paclitaxel-carboplatin is a well-tolerated regimen with promising efficacy as NACT in cervical cancer.

\section{REFERENCES}

1. Sardi, et al. Neoadjuvant chemotherapy in cervical carcinoma stage IIB: a randomized controlled trial. Int J Gynecol Cancer. 1998;8:441-450.

2. Benedetti-Panici P, Greggi S, Colombo A, et al. Neoadjuvant chemotherapy and radical surgery versus exclusive radiotherapy in locally advanced squamous cell cervical cancer: results from the Italian multicenter randomized study. J Clin Oncol. 2002;20:179-188.

3. Rydzewska L, Tierney J, Vale CL, et al. Neoadjuvant chemotherapy plus surgery versus surgery for cervical cancer. Cochrane Database Syst Rev. 2012;12:CD007406.

4. Neoadjuvant Chemotherapy for Locally Advanced Cervical Cancer Meta-analysis Collaboration. Neoadjuvant chemotherapy for locally advanced cervical cancer: a systematic review and meta-analysis of individual patient data from 21 randomised trials. Eur J Cancer. 2003;39:2470-2486.

5. Green JA, Kirwan JM, Tierney JF, et al. Survival and recurrence after concomitant chemotherapy and radiotherapy for cancer of the uterine cervix: a systematic review and meta-analysis. Lancet. 2001;358:781-786.

6. Leone B, Vallejo C, Perez J, et al. Ifosfamide and cisplatin as neoadjuvant chemotherapy for advanced cervical carcinoma. Am J Clin Oncol. 1996;19:132-135.

7. Rose PG, Piver MS, Malfetano JH, et al. A phase II study of weekly cisplatin followed by cisplatin and ifosfamide in advanced or recurrent cervical carcinoma. Cancer. 1993;71:2245-2249.

8. Bonomi P, Blessing JA, Stehman FB, et al. Randomized trial of three cisplatin dose schedules in squamous-cell carcinoma of the cervix: a Gynecologic Oncology Group study. J Clin Oncol. 1985;3:1079-1085.

9. Ferrandina G, Distefano MG, De Vincenzo R, et al. Paclitaxel, epirubicin, and cisplatin (TEP) regimen as neoadjuvant treatment in locally advanced cervical cancer: long-term results. Gynecol Oncol. 2013;128:518-523.

10. Buda A, Fossati R, Colombo N, et al. Randomized trial of neoadjuvant chemotherapy comparing paclitaxel, ifosfamide, and cisplatin with ifosfamide and cisplatin followed by radical surgery in patients with locally advanced squamous cell cervical carcinoma: the SNAP01 (Studio Neo-Adjuvante Portio) Italian Collaborative Study. J Clin Oncol. 2005;23:4137-4145.

11. Lissoni AA, Colombo N, Pellegrino A, et al. A phase II, randomized trial of neo-adjuvant chemotherapy comparing a three-drug combination of paclitaxel, ifosfamide, and cisplatin (TIP) versus paclitaxel and cisplatin (TP) followed by radical surgery in patients with locally advanced squamous cell cervical carcinoma: the SNAP-02 Italian Collaborative Study. Ann Oncol. 2009;20:660-665.

12. Kitagawa R, Katsumata N, Shibata T, et al. Paclitaxel plus carboplatin versus paclitaxel plus cisplatin in metastatic or recurrent cervical cancer: the open-label randomized phase III trial JCOG0505. J Clin Oncol. 2015;33:2129-2135.

13. Mori T, Hosokawa K, Sawada M, et al. Neoadjuvant weekly carboplatin and paclitaxel followed by radical hysterectomy for locally advanced cervical cancer: long-term results. Int $J$ Gynecol Cancer. 2010;20:611-616.

14. Torfs S, Cadron I, Amant F, et al. Evaluation of paclitaxel/ carboplatin in a dose dense or weekly regimen in 66 patients with recurrent or primary metastatic cervical cancer. Eur $J$ Cancer. 2012;48:1332-1340.

15. Eisenhauer EA, Therasse P, Bogaerts J, et al. New Response Evaluation Criteria in Solid Tumours: revised RECIST guideline (version 1.1). Eur J Cancer. 2009;45:228-247.

16. Cancer Therapy Evaluation Program, Common Terminology Criteria for Adverse Events, Version 3.0, DCTD, NCI, NIH, DHHS. March 31, 2003. Available at: http://ctep.cancer.gov. Accessed August 9, 2006.

17. Duenas-Gonzalez A, López-Graniel C, González-Enciso A, et al. A phase II study of multimodality treatment for locally advanced cervical cancer: neoadjuvant carboplatin and paclitaxel followed by radical hysterectomy and adjuvant cisplatin chemoradiation. Ann Oncol. 2003;14:1278-1284.

18. Scandurra G, Scibilia G, Banna GL, et al. Efficacy and tolerability of paclitaxel, ifosfamide, and cisplatin as a neoadjuvant chemotherapy in locally advanced cervical carcinoma. J Gynecol Oncol. 2015;26:118-124. 